\title{
Dispersion in a stratified benthic boundary layer
}

\author{
By LARS RAHM and URBAN SVENSSON, Swedish Meteorological and Hydrological Institute, \\ S-601 76 Norrköping, Sweden
}

(Manuscript received 12 March 1987; in final form 21 April 1988)

\begin{abstract}
A dispersion model for the stratified benthic boundary layer is formulated. It is based on "small-scale" vertical dispersion and a "large-scale" horizontal flow field. A modified Langevin equation governs the stochastic vertical migration of an ensemble of marked fluid elements. These elements are spread out by the horizontal flow, determined by a onedimensional model, which includes a two-equation $(k-\varepsilon)$ turbulence scheme. The later yields statistical information necessary for the stochastic process. Statistical properties of the dispersion process are then calculated from the evolution of the ensemble of elements. A rather idealized case with a linearly stratified fluid subject to a suddenly imposed barotropic pressure gradient is considered. A quasi-geostrophic interior flow is formed with a benthic boundary layer at the bottom. Marked fluid elements are released at the bottom and then followed for several pendulum days. It is found that the dispersion process is well characterized by $K=C u_{*} l$ (where $u_{*}$ and $l$ are the friction velocity at the bottom and the layer thickness, respectively), and where $C \approx 15$. A similar relation but based on external parameters only, becomes:

$\mathrm{K}=\frac{C_{\mathrm{b}}|\partial \boldsymbol{P} / \partial y|^{2}}{\rho^{2} f^{5 / 2} N^{1 / 2}}$,

where $C_{\mathrm{b}} \approx 0.11$ in the range $N / f=28-88$.
\end{abstract}

\section{Introduction}

The benthic boundary layer (BBL) is the fluid layer adjacent to the deep-sea bottom. It is usually characterized by its strong shear and intense vertical mixing; consequently, it usually appears as a well-mixed layer in an otherwise stably stratified environment. The interest in bottom-released material and its dispersal through this layer is to some extent motivated by the use of the seafloor and the underlying geological formations as a repository for disposal of wastes, such as radioactive material etc. Furthermore, the spreading of many pollutants occur when they are attached to "colloidal" particles etc. Hence, the dynamics of the spreading process close to the bottom is of interest from several points of view.

In the past the BBL has been modelled as an Ekman layer with eddy viscosity coefficients chosen to agree with some physical property as e.g., boundary layer thickness. Richards (1982), on the other hand, has presented an alternative model based on a second-order closure scheme similar to the one by Gibson and Launder (1978). This type of model yields both eddy diffusity and boundary-layer thickness. Further, it gives more realistic velocity and veering distributions than the previous models, and it also shows a more satisfactory aggreement with observations. Richards (1982) used this one-dimensional model assuming a horizontal homogeneity in density and flow fields. His model yielded both turbulent mixing, velocity distribution and evolution of the BBL thickness. However, measurements by, among others, Armi and D'Asaro (1980) show substantial inhomogeneities in the BBL on length scales of tens of kilometres. Not only does the BBL's thickness vary in the horizontal plane, but also its density, sometimes leading to a detach- 
ment of the layer. Richards (1984) has therefore studied these and other processes, especially the interaction of mesoscale eddies with the BBL, using a quasi-geostrophic three-layer model.

The aim of the present work is to derive a dispersion model for the BBL in order to study some of those processes active in a real BBLdispersion process. Though the importance of such effects as Ekan pumping, BBL detachment and internal wave breaking are readily recognized, the present model is restricted to describe the spreading of marked fluid elements in a horizontally homogeneous BBL. This, partly because it becomes more tractable and partly because the dispersion process is still valid on short time and length scales, something that may be relevant for estuaries like the Baltic Sea.

The turbulence $(k-\varepsilon)$ model, the two momentum equations and the salinity equation yield the horizontal velocity field of the BBL, while the small-scale vertical migration of marked fluid elements is governed by a Markov process (see the seminal work by Obukhov, (1959)), based on a modified Langevin's equation. The latter is constituted by a random acceleration and a linear drag. The dispersion model is based on shearflow dispersion (Taylor, 1954), which is formed by a vertical shear and small scale vertical mixing. (For a thorough review see Csanady, (1973)). Statistics of an ensemble of marked fluid elements yield the characteristics of the dispersion process. Hence, the particles are followed in a Langrangian frame of reference while the $k-\varepsilon$ model represents an Eulerian system. This dichotomy is circumvented by some empirical relations. The effect of inhomogeneous turbulence is incorporated into the model in accordance with Legg and Raupach (1982). Unfortunately, this modification is only applicable to moderate inhomogeneities, as has been shown by Thomson (1984). The suggested remedy for this failure includes non-Gaussian random acceleration of the fluid elements and hence a drastic increase in the complexity of the model. Consequently the present model has only a limited range of validity. However, a similar model applied to the homogeneous ocean surface layer has been used by Rahm and Svensson (1986) with good results in an investigation of dispersion in a transient Ekman layer. Furthermore, the present model is extended to handle stratification. Hence, the model experiments will yield both the BBL dynamics and gross features of the shear dispersion process in the same layer.

\section{Boundary layer equations}

The flow field is determined by the previously mentioned $k-\varepsilon$ model given by, for example, Svensson (1979), and here in an extended version including buoyancy effects (Svensson, 1980). The momentum equations become, obeying the Boussinesq approximation:

$$
\frac{\partial}{\partial t}\left(\rho_{0} U\right)-f \rho_{0} V=-\frac{\partial p}{\partial x}+\frac{\partial}{\partial z}\left[\frac{\mu_{\mathrm{eff}}}{\rho_{0}} \frac{\partial}{\partial z}\left(\rho_{0} U\right)\right]
$$

$$
\frac{\partial}{\partial t}\left(\rho_{0} V\right)+f \rho_{0} U=-\frac{\partial p}{\partial y}+\frac{\partial}{\partial z}\left[\frac{\mu_{\mathrm{eff}}}{\rho_{0}} \frac{\partial}{\partial z}\left(\rho_{0} V\right)\right],
$$

where $U, V$ and $\rho_{0}$ denote the horizontal velocity components and the reference density respectively. Further, $p$ and $f$ represent the pressure and the Coriolis parameter. Since the fluid is assumed stratified by salt, (this due to its primary application area, the Baltic Sea) the salt concentration equation becomes:

$$
\frac{\partial S}{\partial t}=\frac{\partial}{\partial z}\left[\frac{\mu_{\mathrm{eff}}}{\rho_{0} \sigma_{\mathrm{eff}}} \frac{\partial}{\partial z}(S)\right],
$$

though an analogous treatment of temperature is, of course, possible. The equation of state turns out as:

$$
\rho=\rho_{0}\left(1+\alpha_{\mathrm{s}} S\right)
$$

where $\rho$ is the density and $\alpha_{s}$ represents the coefficient of expansion. $\mu_{\text {eff }}$ is the sum of the turbulent viscosity $\mu_{\mathrm{T}}$ and its laminar counterpart $\mu$;

$$
\frac{\mu_{\mathrm{eff}}}{\sigma_{\mathrm{eff}}}=\frac{\mu}{\sigma}+\frac{\mu_{\mathrm{T}}}{\sigma_{\mathrm{Ts}}},
$$

where $\sigma, \sigma_{\mathrm{Ts}}$ and $\sigma_{\mathrm{eff}}$ are the laminar, turbulent and effective Prandtl/Schmidt numbers respectively.

The turbulent eddy viscosity is calculated from the Kolmogorov/Prandtl relation:

$\mu_{\mathrm{T}}=C_{\mu} \rho_{0} \frac{k^{2}}{\varepsilon}$, 
where $C_{\mu}$ is an empirical coefficient and $k$ and $\varepsilon$ denote the turbulent kinetic energy and its dissipation rate. The turbulent kinetic energy equation reads (see Svensson, 1980):

$$
\begin{aligned}
& \frac{\partial k}{\hat{c} t}=\frac{\hat{c}}{\hat{c} z} {\left[\frac{\mu_{\mathrm{eff}}}{\rho_{0} \sigma_{\mathrm{k}}} \frac{\hat{c} k}{\hat{c} z}\right] } \\
&+\frac{\mu_{\mathrm{T}}}{\rho_{0}}\left[\left(\frac{\hat{c} U}{\hat{c} z}\right)^{2}+\left(\frac{\hat{c} V}{\hat{c} z}\right)^{2}\right]-\varepsilon+P_{\mathrm{b}},
\end{aligned}
$$

while its dissipation rate is given by:

$$
\begin{aligned}
\frac{\hat{c} \varepsilon}{\partial t}=\frac{\hat{c}}{\hat{c} z} & {\left[\frac{\mu_{\mathrm{eff}}}{\rho_{0} \sigma_{\varepsilon}} \frac{\hat{c} \varepsilon}{\hat{c} z}\right] } \\
& +C_{1_{1}} \frac{\mu_{\mathrm{T}}}{\rho_{0}} \frac{\varepsilon}{k}\left[\left(\frac{\hat{c} U}{\hat{c} z}\right)^{2}+\left(\frac{\hat{c} V}{\hat{c} z}\right)^{2}\right] \\
& -C_{2_{r}} \frac{\varepsilon^{2}}{k}+C_{3_{k}} \frac{\varepsilon}{k} P_{\mathrm{b}},
\end{aligned}
$$

where $P_{\mathrm{b}}$ denotes the bouyancy production:

$P_{\mathrm{b}}=\frac{\mu_{\mathrm{T}}}{\rho_{0}}\left[\frac{g \alpha_{\mathrm{s}}}{\sigma_{\mathrm{Ts}}} \frac{i S}{\hat{c} z}\right]$

The turbulent Prandtl/Schmidt numbers, $\sigma_{i}$ and $\sigma_{k}$, as well as the empirical coefficients $C_{t_{i}}, C_{2_{i}}$ and $C_{3_{t}}$ and the Prandtl/Schmidt number of salt, $\sigma_{\mathrm{Ts}}$, are found in Table 1. A wide variety of experiments makes the foundation of these coefficients (Rodi, 1980). This set of equations is solved numerically in its finite difference form by an implicit scheme and a standard tri-diagonal matrix algorithm. These equations and solutions have been discussed extensively in the past, hence the reader is referred to Svensson (1978, 1980) for further details. A validation of the turbulence model in the context of a stratified surface layer with experimental data has been carried out in Omstedt, Sahlberg and Svensson (1983) with good results.

The present boundary conditions are represented by a no-slip condition at the rigid bottom of given roughness length $Z_{0}$ (in the following calculations $Z_{0}=510^{-3} \mathrm{~m}$ in order to represent a rather smooth bottom), while the top condition is one of free slip. The initially quiescent fluid is forced by a prescribed constant pressure gradient. This in order to simplify the investigations. The boundary conditions for $k$ and $\varepsilon$ are obtained from the assumption of local equilibrium between production and dissipation of kinetic energy (Rodi, 1980). Finally, zero density-flux conditions are prescribed both at the top and the bottom.

The boundary layer may be more or less turbulent depending upon the bottom roughness. This behaviour is reflected in the "roughness Reynolds number" $\tilde{R}_{\mathrm{e}}=h_{*} u_{*} v^{-1}$, where $h_{*}$ and $u_{*}$ are the rms surface height $\left(h_{*} \approx 30 Z_{0}\right)$ and the friction velocity respectively. If $\tilde{R}_{\mathrm{e}}<5$, the flow in the viscous sublayer is hydrodynamically smooth, but for $\tilde{R}_{\mathrm{e}}>30$ it becomes rough (Bowden, 1978). In the former case, the effects of viscosity dominate over the Reynolds stresses, while in the latter case, the roughness elements themselves generate turbulent wakes, which are responsible for an inviscid drag at the surface. (For details, see Tennekes and Lumley, 1972.)

The present $k-\varepsilon$ model uses prescribed values of $k$ and $\varepsilon$ at the bottom, which are related to the calculated shear stress (Launder and Spalding, 1974). These calculations are based on the assumption that the first cell is considerably deeper than the characteristic length scale of the viscous sublayer, $v u_{*}{ }^{-1}$ (i.e., in the range $\left.(30-100) v u_{*}{ }^{-1}\right)$. Hence, the velocity and energy distributions within the viscous sublayer cannot be resolved in the present model. Consequently, this will lead to an overestimation of the transfer process in the region close to the bottom. However, the Markov process itself is still valid deep into the viscous sublayer, even for low $\tilde{R}_{\mathrm{e}}$. This because of the random motions, which are too insignificant to influence the Reynold stresses, but still can transfer solutes more efficiently than their molecular diffusivities may indicate. The bottom molecular diffusivity will

Table 1. Constants used in the calculations

\begin{tabular}{llllllllll}
\hline$\sigma_{\mathrm{1s}}$ & $\sigma_{\mathrm{k}}$ & $\sigma_{i}$ & $C_{\mathrm{I}_{i}}$ & $C_{z_{i}}$ & $C_{z_{i}}$ & $C_{\mu}$ & $C_{\sigma}$ & $C_{\mathrm{r}}$ & \\
\hline 1.0 & 1.4 & 1.3 & 1.44 & 1.9 & 0.8 & 0.09 & 0.3 & 0.2 \\
\hline
\end{tabular}


only dominate the transfer process in an extreme neighbourhood of the bottom (Chriss and Caldwell, 1984).

To summarize the above, the $k-\varepsilon$ model seems able to yield both velocity distribution and statistical information of the flor

\section{Dispersion model}

The dispersion model used in this work is similar to the one presented in Rahm and Svensson (1986), hence only its basic features will be outlined below.

The vertical diffusion of a dissolved substance is modelled by a single-particle Markov process, based on a modified Langevin's equation:

$\frac{\partial w}{\partial t}=\lambda \xi-\alpha w+F$,

which is formed by a random acceleration, $\lambda \xi$, (since the time scale of the molecular collision is much smaller than that of the turbulence, the Kolmogorov time scale) and a linear drag, $\alpha w$. $w$ and $\xi$ represent the vertical velocity of a marked fluid element and a statistically stationary stochastic (Gaussian) process respectively. Legg and Raupach (1982) introduced a correction term, $F$, because the unmodified Langevin's equation yielded a spurious behaviour in cases with non-homogeneous turbulence. A drift of particles took place along the gradient of vertical velocity variance, $\sigma_{w}^{2}$ (i.e., toward regions of low tubulence intensity). This gradient is, however, associated with a pressure gradient (Hinze, 1975); consequently this term is incorporated into Langevin's equation. The justification of this has been investigated by Thomson (1984), who found that it is valid only for a moderate inhomogeneity in velocity variance, i.e., if (in accordence with Thomson, 1984):

$\left|\frac{\tau}{\sigma_{w}} \frac{\partial \sigma_{w}^{2}}{\partial z}\right| \ll 1$.

Here, $\tau$ represents the typical time-scale of motion. Thomson (1984) showed that for stronger inhomogeneities a non-Gaussian random acceleration yielded a satisfactory behaviour. Since only a moderate inhomogeneity in turbulence is assumed in this study, the approach of Legg and Raupach (1982) will still be used.
Eq. (3.1) is formulated within a finitedifference context, thus forming a Markov sequence:

$w_{n+1}=a_{n} w_{n}+b_{n} \sigma_{w_{n}}+c_{n}$,

where index $n$ denotes the time-step. The coefficients are defined in accordance to Legg and Raupach (1982):

$a_{n}=\mathrm{e}^{-\Delta t \cdot \tau_{r_{n}}}$,

$b_{n}=\left(1-\mathrm{a}_{n}^{2}\right)^{1 / 2}$,

$c_{n}=\tau_{l_{n}} \frac{\hat{c}}{\hat{c} z}\left(\sigma_{w_{n}^{2}}^{2}\right)\left(1-a_{n}\right)$,

where $\tau_{l_{n}}$ is the Lagrangian autocorrelation time scale. Thus each particle has a "memory" of its turbulent motion. The Markov-chain yields the vertical velocity of the particle, given $\tau_{l_{n}}$ and $\sigma_{w_{n}}$. The time-step $\Delta t$ must satisfy the following inequality:

$\tau_{\lambda} \ll \Delta t \ll \tau_{l}$,

where $\tau_{j}$ denotes the Taylor microscale.

The $k-\varepsilon$ model yields characteristic turbulent velocity and time scales in an Eularian frame of reference (Rahm and Svensson, 1986):

$\tau_{\mathrm{E}}=C_{\tau} \frac{k}{\varepsilon}$,

$\sigma_{w_{\mathrm{F}}}^{2}=\mathrm{C}_{\sigma} k$,

where $C_{\mathrm{r}}$ or $C_{\sigma}$ are empirically determined coefficients (see Table 1). No straightforward relation exists between Eulerian and Langrangian "statistics" (Pasquill and Smith, 1983), but empirical relations have been found (see also Rahm and Svensson, 1986):

$\tau_{l}=\frac{C_{\mu}}{C_{\sigma}} \frac{k}{\delta}$,

$\sigma_{w}^{2}=\mathrm{C}_{\sigma} k$.

They are based on the equivalence of both the velocity variance (Hanna, 1982) and the effective exchange coefficient (Batchelor, 1949). Finally, the Taylor microscale may be estimated (according to Tennekes and Lumley, 1972) by:

$\tau_{i}=(15 v / \varepsilon)^{12}$

The parameters are calculated for each time step and grid point by the $k-\varepsilon$ model thus 
enabling a treatment of even transient problems. Since the time step in the Markov process is quite small compared to that required in the $k-\varepsilon$ model, $\sigma_{\mathrm{w}}$ and $\tau$, are assumed constant over each time step in the turbulence model.

\section{Benthic boundary layer}

An expanding grid is used (where the control volumes are proportional to a geometrical series: $\Delta z_{i+1} / \Delta z_{i}=1.1$, with the highest resolution at the bottom, since the interest is focused on the bottom boundary layer). A depth of $H=30 \mathrm{~m}$ is assumed sufficient to allow for the formation of a quasi-geostrophic interior due to the prescribed barotropic pressure gradient. (Its strength is 5 $10^{-3} \mathrm{~N} / \mathrm{m}^{2}$.) The time-step of the hydrodynamical model is $600 \mathrm{~s}$ in order to yield a time-step independent solution, while that of the Markov process is fixed to $10 \mathrm{~s}$. The latter in order to resolve the strong gradients appearing in the boundary layer, as both $\tau_{\text {, and }} \tau_{\dot{i}}$ become small in this region. However, because of the constant time-step, the inequality (3.5) is not fulfilled everywhere. The errors induced are nevertheless assumed limited, since $\Delta t \ll \tau$, in most parts of the region. (For further details, see Rahm and Svensson, 1986.)

First, a neutrally stratified BBL is assumed in order to illustrate the behaviour of the model. Typical profiles of the velocity components and the angle of the flow from its interior direction are presented in Fig. 1 for a case with a bottom surface Rossby number $R_{\mathrm{o}}=\bar{U} / Z_{0} f=7 \cdot 10^{4}$. Here $\bar{U}$ denotes a typical velocity of the geostrophic flow. The general characteristics of a neutrally stratified boundary layer flow is obtained with this turbulence model.

The mixing length is assumed to have an asymptotic value at large distances from the boundary in some second-order closure models. This value is assumed to be proportional to the scale height of turbulence intensity. Mofjeld and Lavelle (1984) found in an investigation of unstratified bottom boundary layers that the optimal value of the mixing length parameter $\gamma$ was approximately $0.2-0.3$ for oceanic conditions.

The asymptotic mixing length $l_{0}$ may be

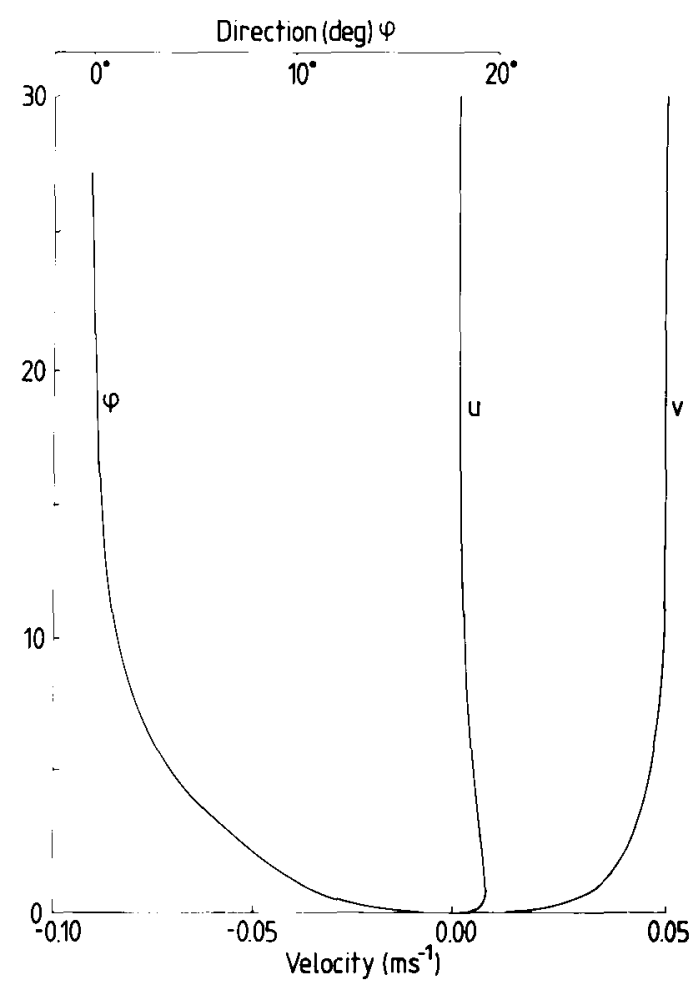

Fig. I. Velocity distribution $(u, v)$ at $t=46.7 \mathrm{~h}$ for a case with $N / f=0$, which is forced by a pressure gradient of $5 \cdot 10^{-3} \mathrm{~N} / \mathrm{m}^{2}$. The deviation of the flow from its "interior" value, $\phi$, is also shown.

written (cf., Mofjeld and Lavelle, 1984):

$l_{0} \approx \gamma^{2} u_{*} f^{-1}$,

where $u_{*} f^{-1}$ represents the similarity height. Hence, $\gamma$ may be calculated from the distribution of non-dimensionalized characteristic turbulent scales:

$\gamma^{2} \approx \sigma_{\mathrm{w}_{\mathrm{F}}} \tau_{\mathrm{F}} f u_{*}^{-1}=C_{\mathrm{\tau}} C_{\mathrm{I} / 2}^{\sigma} k^{3 / 2} \varepsilon^{-1} f u_{*}^{-1}$.

The distribution of the Lagrangian integral length scale (cf., Tennekes and Lumley, 1972):

$l_{l}=C_{\mu} C_{\sigma}^{-1 / 2} k^{3 / 2} \varepsilon^{-1}$,

and the corresponding calculated $\gamma$ is shown in Fig. 2 for some different cases.

The value for the unstratified case $(\gamma \approx 0.30)$, which has been used in several models according to Mofjeld and Lavelle (1984), lies in the upper end of their interval estimated for oceanic 


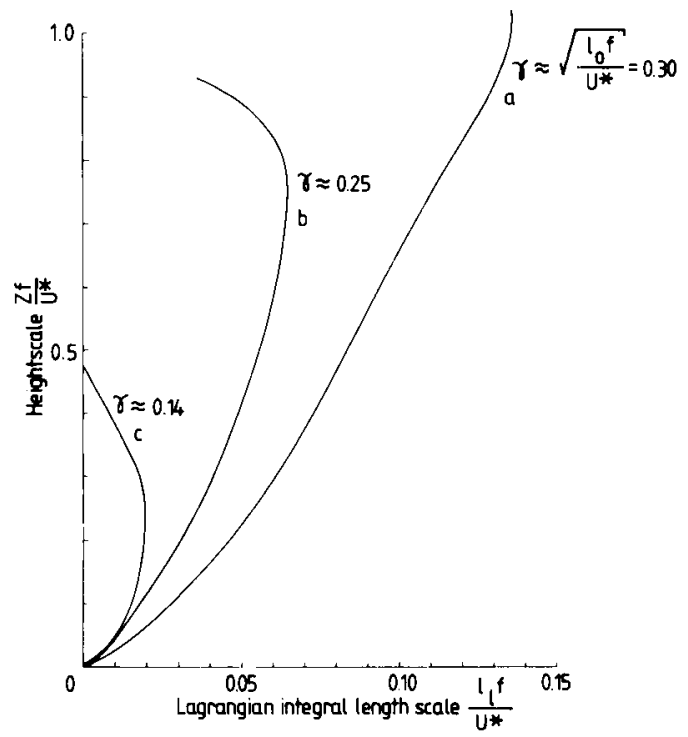

Fig. 2. The non-dimensional Lagrangian integral length scale $l_{i} f u_{*}^{-1}$ versus height $z f u_{*}^{-1}$ for (a) unstratified case $N / f=0$, (b) stratified case $N / f=18$ and a "frozen" profile, and (c) same as in (b) but for a "stationary case". The mixing length parameter $\gamma$ is shown for each case.

conditions. However, this situation is drastically changed in a stably stratified fluid. Even a weak, initially linear stratification $(N / f=18$, where $N$ is the bouyancy frequency) will substantially reduce the parameter values, as is shown in Fig. 2, for both a "frozen" profile $(\gamma \approx 0.14)$ and for a welldeveloped benthic boundary layer $(\gamma \approx 0.25)$. Since this stratification is close to the accuracy of oceanic field data, one can conclude that the results obtained by the present $k-\varepsilon$ model seem to agree well with those estimated by Mofjeld and Lavelle (1984). The length scales produced by this model are in consort with those found in a geophysical context, despite that it is "calibrated" against laboratory measurements.

An ensemble of marked fluid elements (particles) were released at the bottom after the system had been allowed to spin up for one pendulum day. The evolution of this cloud is shown in Fig. 3a, where the relative concentration of particles are presented in a horizontal projection for every half a pendulum day. It is evident that the cluster is both advected by the "Ekman transport" and spread by the velocity
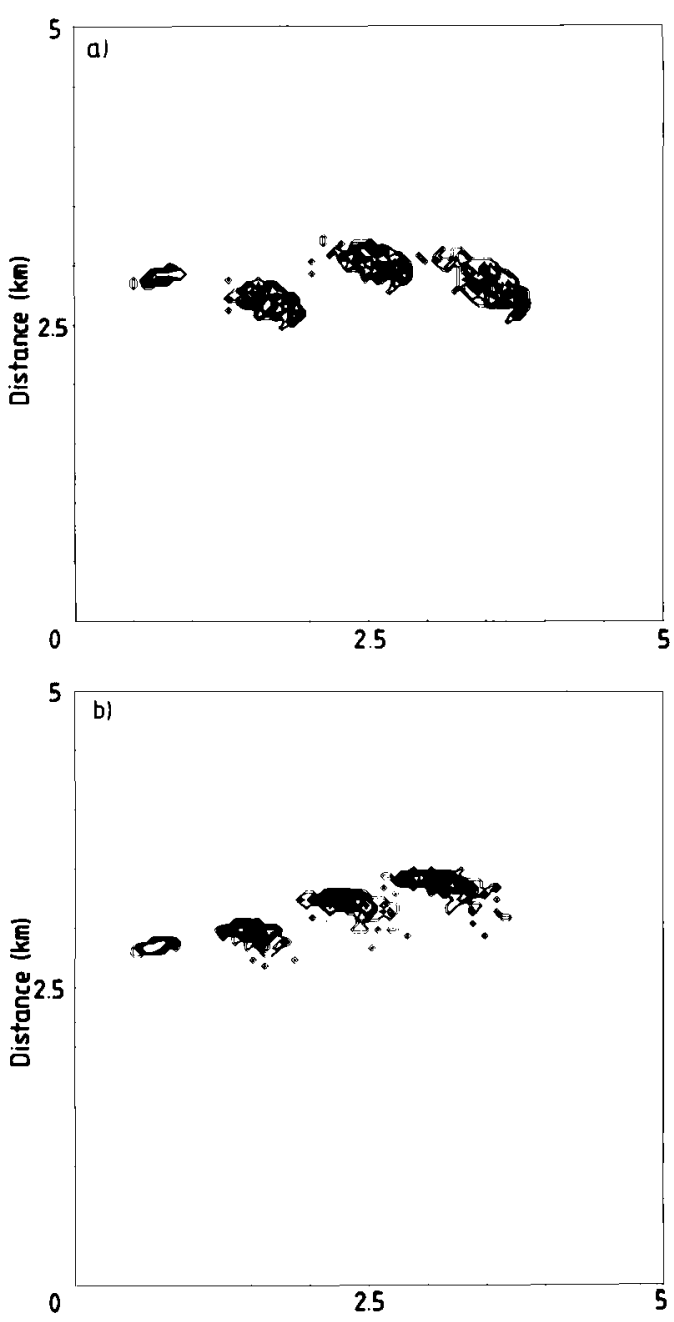

Fig. 3. Relative concentration plot with a contour interval of $0.2 \cdot 10^{-2}$ based on an ensemble of 400 particles released at $x=0 ; y=2.5 \mathrm{~km}$ after every half a pendulum day for: (a) $N / f=0$ and (b) $N / f=56$.

shear. (Note that the particles are not subject to any diffusive process in the horizontal plane.)

The variance of the ensemble was calculated in the two principal directions, along the applied pressure gradient $(y)$ and orthogonal to it $(x)$. A number of 200 particles was used to ensure a reasonable estimate of the variances. A measure of the "total" variance was defined as (cf., Okubo, 1971):

$\sigma_{\mathrm{rc}}^{2}=2 \sigma_{x} \sigma_{y}$ 


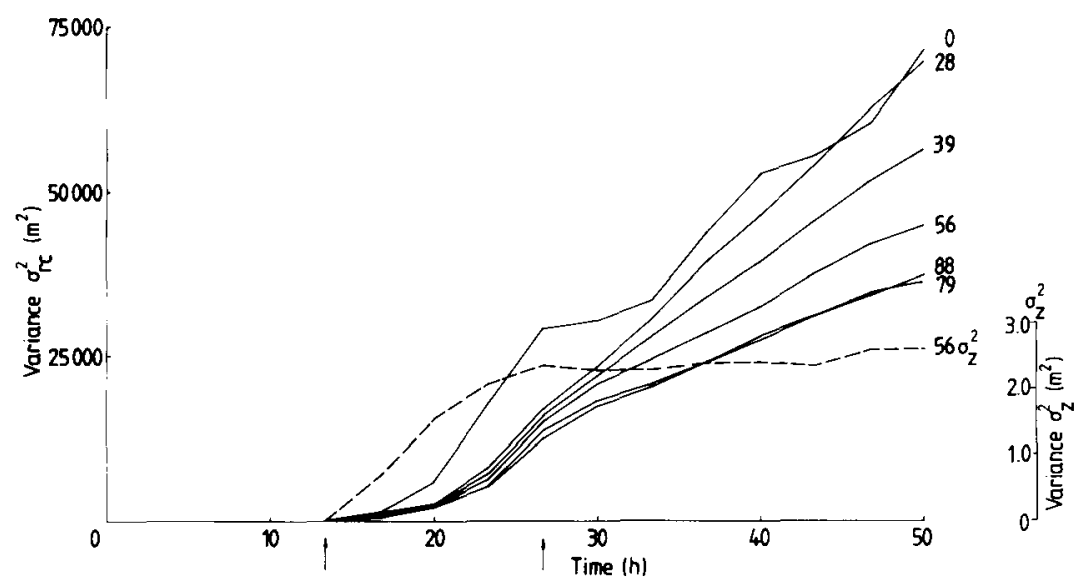

Fig. 4. The variance $\sigma_{\mathrm{rc}}^{2}$ versus time for $N / f=0-88$. The evolution of $\sigma_{z}^{2}$ with time is also shown but only for $N / f=56$ and for one realization. The arrows indicate one "pendulum day".

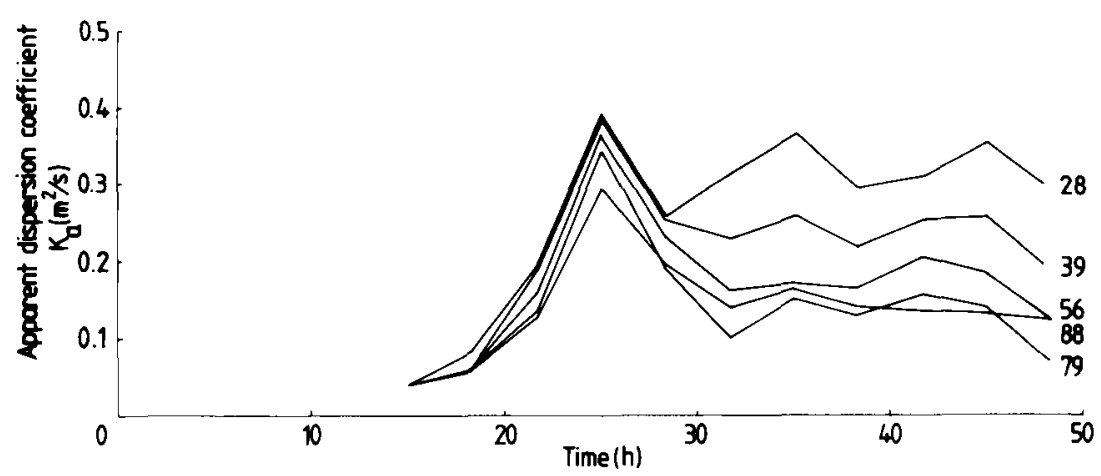

Fig. 5. The apparent diffusivity versus time for $N / f=0-88$.

The evolution of $\sigma_{\mathrm{rc}}^{2}$ with time is presented in Fig. 4, while the corresponding "apparent" diffusivity (cf. Csanady, 1973),

$K_{\mathrm{a}}=\frac{1}{2} \frac{\hat{c}}{\partial t}\left(\sigma_{\mathrm{rr}}^{2}\right)$

is shown in Fig. 5.

\section{Stratified boundary layer}

Consider, for simplicity, an initially linearly stably stratified fluid at rest, that is suddenly subjected to a barotropic pressure gradient. This gives rise to a quasi-geostrophic interior flow and strong vertical velocity gradients at the bottom, which leads to an enhanced turbulence production and consequently to the development of a well-mixed BBL, see Fig. 6. The variation of layer thickness with time is shown in Fig. $7 \mathrm{a}$ and b for some different salinity gradients. The growth rate decreases with increasing stratification. The height is rather arbitrarily defined as the height $h_{\mathrm{b}}$, at which $\mu_{\text {eff }}$ equals its double laminar value;

$h_{\mathrm{b}}=z\left(\mu_{\mathrm{T}}=2 \mu\right)$.

The evolution of the layer thickness is qualitatively in agreement with those reported by Pollard et al. (1973) for the oceanic surface layer, though the actual values obtained by the present model is roughly a factor 2 greater than theirs, 


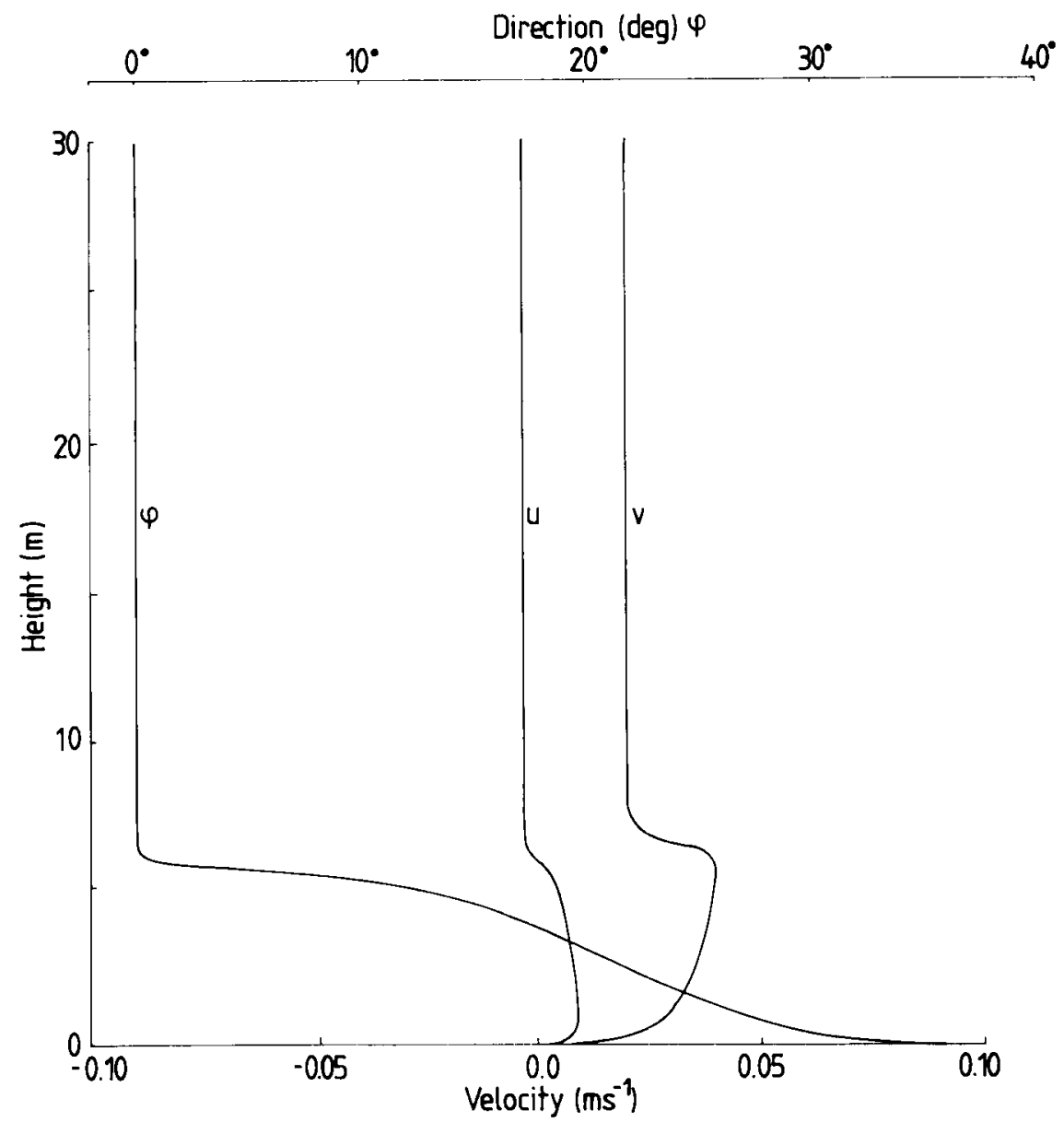

Fig. 6. Same legend as in Fig. 1 but for $N / f=56$.

partly due to the choice of reference level made above. The BBL rapidly reaches a quasi-steady state after only one pendulum day, though a nontrivial true asymptotic state does not exist in this model due to diffusive effects. The cloud of particles were then released one pendulum day after the onset. The effects of inertial oscillations are clearly observable in the results.

The dispersal of particles will be confined to the BBL, because of the distribution of turbulent kinetic energy (Fig. 8) which decreases with height within the BBL until it reaches the laminar quasi-geostrophic interior.

Both $k$ and $\varepsilon$ diminish in this interior, but due to the numerical limit used, their ratio goes to unity. Hence, the calculations of $\tau_{l}$ become erro- neous. This result is, however, not fatal, as $\sigma_{w}$ decreases in an analogous way to $k$, hence drastically limiting the vertical migration of particles in the "halocline". The distribution of $\tau_{l}, \tau_{\lambda}, \sigma_{\mathrm{w}}$ and the skewness ratio are all shown for $N / f=56$ in Figs. 9 and 10. The problems with time-step in the Markov process also remains in the stratified cases, while the skewness ratio shows that the chosen model is within its bounds of validity.

The evolution of the ensemble of particles with time, as is illustrated in the relative concentration of particles for $N / f=56$, is shown in Fig. 3b (together with its homogeneous counterpart in Fig. 3a). Here the mean horizontal drift as well as the spread is evident. The actual behaviour of the particles is illustrated by a few trajectories in the 

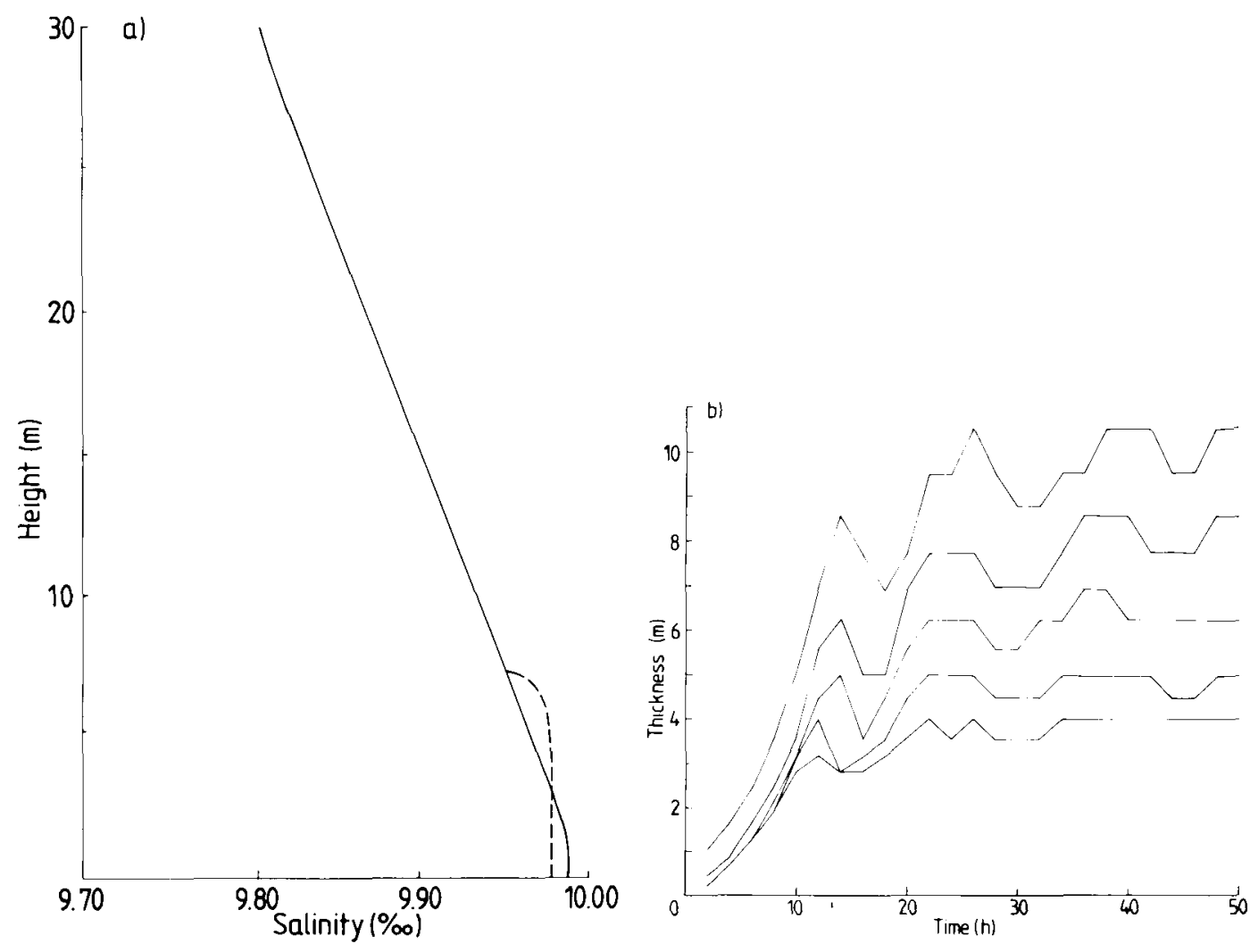

Fig. 7 (a) Salinity profiles for $N / f=56$ at $13.3 \mathrm{~h}$ (solid line) and $46.7 \mathrm{~h}$ (dashed line). (b) The thickness of the benthic boundary layer for one realization and for $N / f=28-88$. The arrow indicates one pendulum day after release.

$x-z$ plane (see Fig. 11). The paths illustrate both their erratic vertical movements and the effect of the shear flow in the BBL.

The vertical spreading of the whole ensemble is illustrated by its vertical variance $\sigma_{z}^{2}$ for the same case as above. This is shown together with $\sigma_{\mathrm{rc}}^{2}$ (for some different stratifications) in Fig. 4. Two distinct phases in cluster growth are discernible. The first phase is characterized by an "accelerated" dispersion process when cloud size grows faster than a linear increase with time. The duration of this phase is roughly one pendulum day, and it also defines the time scale for the particles to reach their stationary distribution in the vertical within the boundaries. The second phase corresponds to a linear growth in $\sigma_{r \mathrm{r}}^{2}$, i.e. the cluster grows more or less as by "Fickian" diffusion. This behaviour is in accordance with the analysis by Staffman (1962) for a case with a bounded fluid.

This transition takes place when the cluster reaches a typical length scale intermediate to the BBL thickness and the scale of inertial oscillations. The calculated dispersion coefficient (4.5) is presented in Fig. 5 for the same cases as in Fig. 4. Not surprising, both $K_{\mathrm{a}}$ and $\sigma_{\mathrm{rc}}^{2}$, decreases with increasing stratification. The oscillations found are due to the inertial oscillations of the fluid.

\section{Discussion}

The simulation of the response of a BBL in an initially continuously stratified fluid subject to a suddenly imposed barotropic pressure gradient yields results qualitatively similar to those reported by Richards (1982). This is not surprising, 


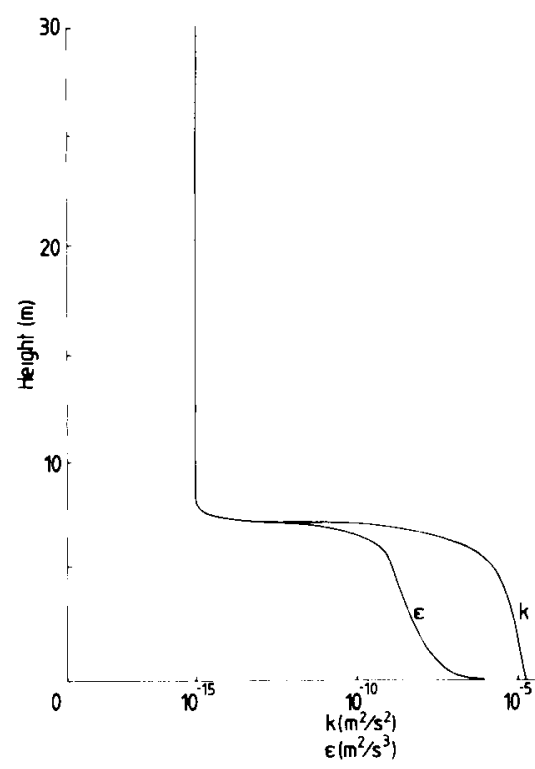

Fig. 8. Turbulent energy distribution $(k)$ and its dissipation rate $(\varepsilon)$ for $N / f=56$. since the present hydrodynamic model is based on a similar type of turbulence model as has been used by Richards (1982). His model has predictive equations for all second order correlation turbulent quantities, while the present model relates the Reynolds stresses to the local mean velocity gradients. The hydrographic situation is, however, somewhat different, as a stronger stratification is chosen to cope with the hydrographic conditions in estuaries like the Baltic Sea. Typical values of $N / f$ are in the range $20-90$. The model results are, of course, sensitive to some of the empirical coefficients chosen (see Table 1), but the set used is the optimal one for a wide range of flows (Rodi, 1980), and hence they are not altered in this experiment.

The formation of a well-mixed BBL and its development has direct consequences for the dispersion process. The particles become "trapped" between the bottom and the "halocline" a the top of the BBL. This trapping (which to some extent is caused by the idealized

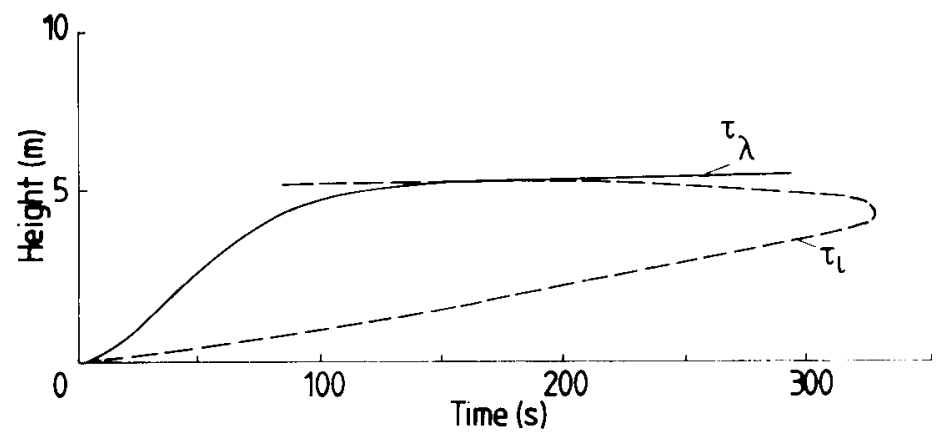

Fig. 9. Distributions of the Lagrangian time scale $\tau_{/}$(dashed line) and the Taylor micro scale $\tau_{\dot{i}}$ (solid line) in the boundary layer for $N / f=56$.

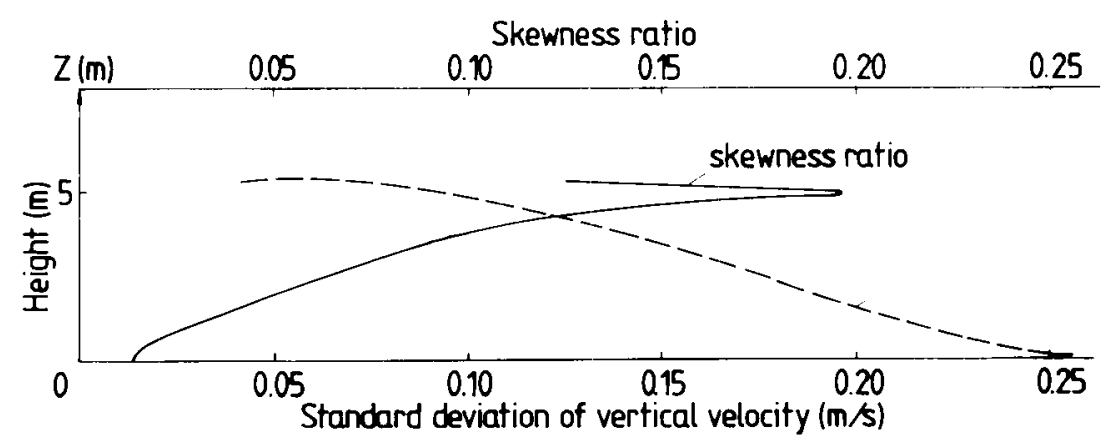

Fig. 10. Distribution of velocity variance $\sigma_{\mathrm{w}}$ (dashed line) and the non-dimensional skewness ratio (solid line) in the boundary layer for $N / f=56$.

Tellus $41 \mathrm{~A}(1989), 2$ 


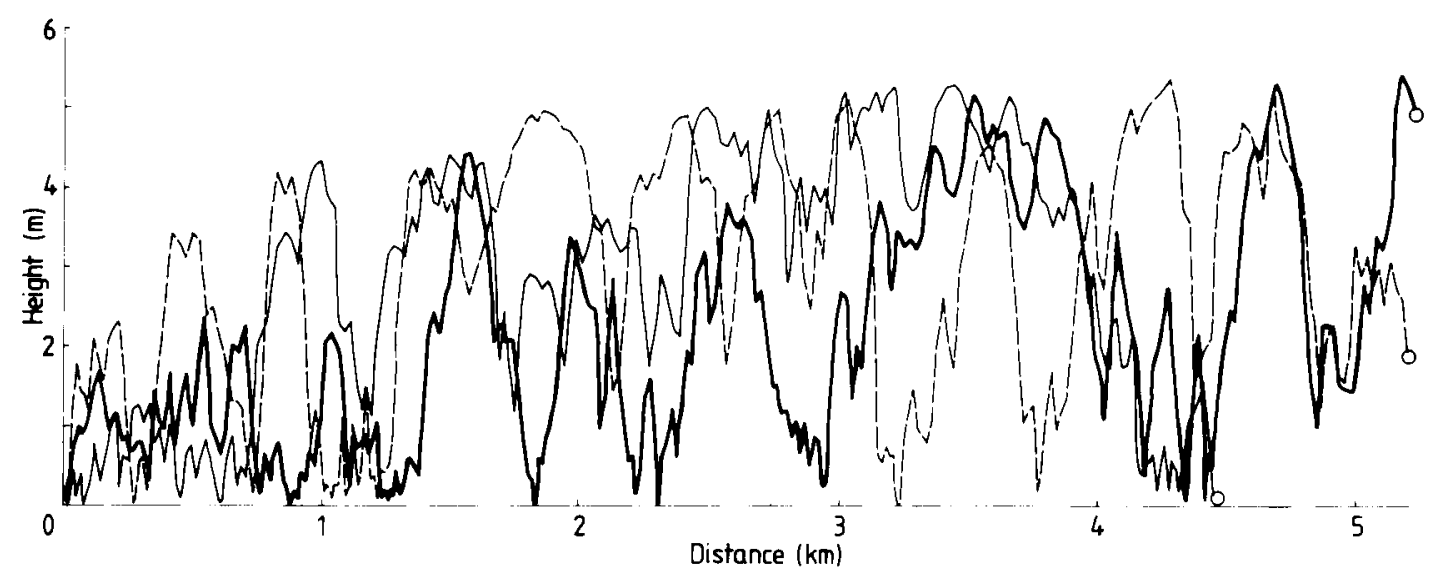

Fig. Il. Trajectories for three different particles for the case $N / f=56$. The circles indicate the end positions $40 \mathrm{~h}$ after release.

forcing) is somewhat in contrast to what is assumed occuring in the real oceans, where a manifold of exchange processes contribute to the dispersion process (see, e.g., Richards, 1985). However, these process are beyond the scope of this one-dimensional model.

The BBL is dominated by small-scale turbulence, which rapidly mixes the fluid vertically. The vertical exchange coefficients fall within the range $10^{-3}-10^{-4} \mathrm{~m} / \mathrm{s}$. This is at the lower end of the estimates found for oceanic conditions (see, e.g., Bowden (1978) or Sarmiento (1978)), something that is not so surprising when considering the present case with a higher degree of stratification and idealized forcing. However, due to the vertical shear within the BBL and the large-scale inertial oscillations, the horizontal dispersion becomes more effective $\left(\sim 10^{-1} \mathrm{~m}^{2} / \mathrm{s}\right)$, though the stratification (and therefore the thickness of BBL) still strongly controls the process.

Note that by releasing the particles after only one pendulum day from a sudden start of forcing, the resulting inertial oscillations will increase the effectivity of the dispersion process by increasing the variability in the forcing of the benthic boundary layer. An increase in time-lag (between the start of forcing and the release of particles) by a factor of seven reduced the variance by $10-$ $15 \%$.

However, a more systematic investigation of the role of the inertial oscillations is beyond the scope of this work, since they depend on such things as the initial conditions, stratification, etc.
Verification of the obtained dispersion rates against field experiments has not been carried out because of a lack of relevant experimental data. However, a comparison against independent estimates of shear flow dispersion has been made. Taylor (1954) presented a relation for this type of dispersion in a pipe, based on only friction velocity and a characteristic length scale $l$ :

$K_{\mathrm{d}}=C u_{*} l$,

where $K_{\mathrm{d}}$ and $C$ represent the coefficients of dispersion and proportionally respectively. Elder (1959) has applied this formulation on a freesurface channel flow with similar results. Further, Bowden (1965) has made similar derivations but now for some different velocity profiles.

By using the model predictions $K_{\mathrm{a}}, u_{*}$ and $h_{\mathrm{b}}$ in eq. (6.1), the proportionality coefficient $C$ is determined for some different stratifications. Though tentative and only based on a single realization for each initial salinity profile, the values of $C$ are calculated for roughly the first three pendulum days of the dispersion process. This process is, for the major part of the period, characterized by a linear growth in $\sigma_{\mathrm{rc}}^{2}$. (This evidently leads to a slight underestimation of $C$ because of the rapid growth of $\sigma_{\mathrm{rc}}^{2}$ in the beginning of the period.) These values are compared with the estimates mentioned above, see Table 2.

A high degree of agreement is found with regard to the different hydrodynamical conditions: the stratified benthic boundary layer versus neutrally stratified, non-rotating channel 
Table 2. Coefficient of proportionality $D$ in the dispersion relation $K_{d}=C u_{*} l$

\begin{tabular}{lll}
\hline Investigator & $C$ & Length scale $/$ \\
\hline $\begin{array}{l}\text { Taylor (1954) } \\
\text { Elder (1959) }\end{array}$ & 5.1 & $=$ diameter of pipe \\
$\begin{array}{l}\text { Bowden (1965) } \\
\text { present results for }\end{array}$ & 6.9 & $=$ channel depth \\
$N / f=28-88$ & 15.3 & $=$ channel depth \\
\end{tabular}

flows. Consequently, it seems that elements from classical hydrodynamic theory are valid even in the present context.

From a practical point of view, the results of the simulations would be most useful. if the "apparent" dispersion coefficient $K$ (see eq. (6.1)) could be estimated in terms of external flow parameters only.

The friction velocity may be obtained from the interior quasi-geostrophic velocity, which is forced by the imposed pressure gradient, $\bar{U} \sim(\rho f)^{-1} \partial P / \hat{C} y$, (cf., Tennekes and Lumley, 1972).

$u_{*} \sim C_{d}^{12}(\rho f)^{-1}\left|\frac{\hat{C} P}{\hat{\partial} y}\right|$,

where $C_{d}$ is the coefficient of bottom friction. The height of the boundary layer, $h$, may be estimated from friction velocity, stratification and rotation (cf., Pollard et al., 1973);

$h \approx 2^{3 / 4} u_{*}(N f)^{-1,2}$.

(The coefficient given is based on field measurements in the wind-mixed surface layer, consequently its numerical value may not be valid for the present layer.)

This yields a functional dependence :

$K=\frac{D|\hat{c} P / \hat{C} y|^{2}}{\rho^{2} f^{52} N^{12}}$

where $D$ is an hitherto unspecified coefficient. Analogous calculations to those above, but now based on the external parameters in (6.4) only, yield the results presented in Fig. 12 (together with the corresponding $K_{\mathrm{a}}$-values). A reasonable choice for the parameter range in question is

$D=0.11$

Eq. (6.1), which includes rotation, stratification and bottom stress, does not explicitly take into

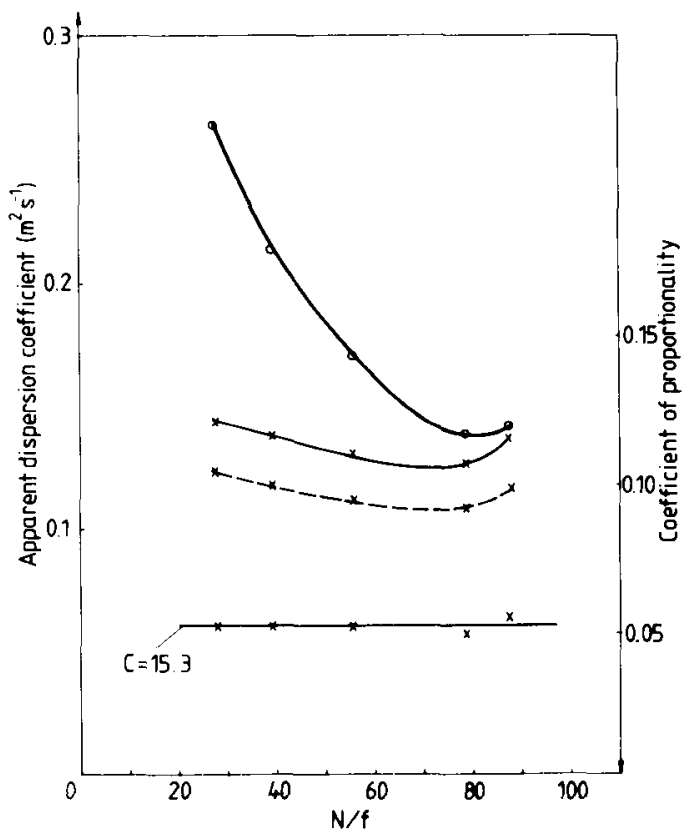

Fig. 12. Apparent dispersion coefficient $K$ and coefficient of proportionality $D$ versus stratification. $D$ is calculated for estimated boundary layer heights by both Pollard et al. (1973) (.) and by the model $(x)$. The straight line indicates the mean value of $C$.

account the shear distribution and veering factors. The overall changes in veering with respect to stratification is, however, small, as seen in Fig. 13a. Consequently it can be included into $\mathrm{D}$. This is partly due to $N / f \gg 1$, and thus the rotation becomes of minor importance (cf. Kitaigorodskii, 1988) in the well-mixed BBL. Though the veering is considerable (see Fig. 13a), a large part of it takes place in regions close to the boundaries, where the turbulent exchange processes (say $\mu_{\mathrm{T}}$, Fig. 13b) are less pronounced. This makes the dispersion process less sensitive to the effect of veering. (A comparison between Fig. 1 and Fig. 13a shows an increase in veering with increased stratification, as is reported by, e.g. Wyngaard (1975), but no systematic investigation has been carried out in this work.)

Future work in this field should address such problems as dispersion in the viscous sublayer and the sedimentation/resuspension processes, areas in which the present Lagrangian approach may be fruitful. The actual dispersion model should be readily applicable to both two- and 

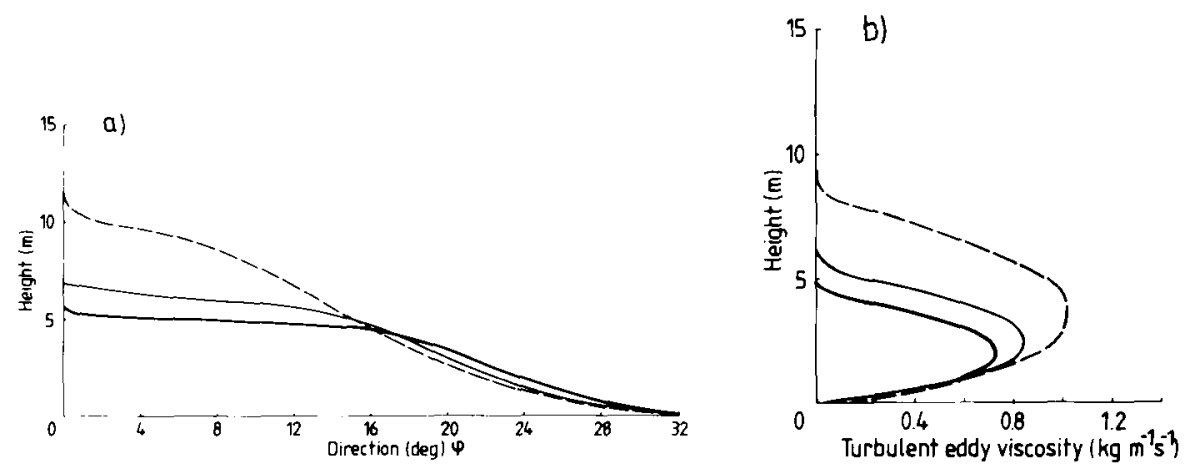

Fig. 13. The effect of stratification upon the veering and turbulent eddy viscosity for three different stratifications, $N / f=28$ (dashed line), $N / f=56$ (thin solid line) and $N / f=77$ (heavy solid line). (a) Flow direction $\phi$ versus height for the three different stratifications. (b) Turbulent vertical eddy viscosity $\mu_{\mathrm{T}}$ versus height for the three different stratifications.

three-dimensional hydrodynamical models (under condition that it gets its statistical turbulence information). This is of interest in applied oceanography, where complex forcing, stratification and topography of ten make the problem in question hardly solvable. The major weakness is its short time-step dictated by the inequality (3.5).

\section{Conclusions}

The following conclusions can be drawn from this work.

The turbulence $(k-\varepsilon)$ model yields a reasonable description of the BBL dynamics.

The Markov-chain model simulates well the characteristic behaviour of the spreading process for a passive tracer in a Langrangian frame of reference. It is released from a point source within the boundary layer. The spreading takes place without any tendency to false accumulation at the boundaries.

Calculated dispersion rates reveal a reasonable agreement with those given in literature for spreading in non-rotating, neutrally stratified channel flows.

An empirical relation for the dispersion coefficient based on only external parameters is found.

\section{Acknowledgements}

The authors appreciate very much the helpful comments of the referees. Furthermore, the authors are also indebted to Mrs. V. Kuylenstierna for valuable help with the preparation of the manuscript. This work was supported by both the Swedish National Sciences Research Council and the National Swedish Environmental Protection Board.

\section{REFERENCES}

Armi, L. and D'Asaro, E. 1980. Flow structures of the benthic ocean. J. Geophis. Res. 85, 469-484.

Batchelor, G. K. 1949. Diffusion in a field of homogeneous turbulence. Aust. J. Res. A2, 437-450.

Bowden, K. F. 1965. Horizontal mixing in the sea due to shearing current. J. Fluid. Mech. 21, 83-95.

Bowden, K. F. 1978. Physical problems of the benthic boundary layer. Geophysical Surveys 3, 255-296.
Chriss, T. M. and Caldwell, D. R. 1984. Universal similarity and the thickness of the viscous sublayer at the ocean floor. J. Geophys. Res. 89, 6403-6414.

Csanady, G. T. 1973. Turbulent diffusion in the environment. Dordrecht: D. Reidel Publ. Co., 248 pp.

Elder, I. W. 1959. The dispersion of marked fluid elements in turbulent shear flow. J. Fluid Mech. 5, 544-560. 
Gibson, M. M. and Launder, B. E. 1978. Ground effects on pressure fluctuations in the atmospheric boundary layer. $J$. Fluid Mech. 86, 491-511.

Hanna, S. R. 1982. Applications in air pollution modelling. In: F. T. M. Nieuwstadt and H. van Dop (eds.) Atmospheric turbulence and air pollution modelling Dordrecht: D. Reidel Publ. Co., 275-310.

Hinze, J. O. 1975. Turbulence (2nd edition). New York: McGraw-Hill, 586 pp.

Kitaigorodskii, S. A., 1988. A note on similarity theory for atmospheric boundary layers in presence of background stable stratification. Tellus 40A, 434-438.

Launder, B. E. and Spalding, D. B. 1974. The numerical computation of turbulent flows. Computer methods in applied mechanics and engineering 3, 269-289.

Legg, B. J. and Raupach, M. R. 1982. Markov chain simulation of particle dispersion in inhomogeneous flows: The mean drift velocity induced by a gradient in Eulerian velocity variance. Boundary-Layer Meteorol. 24, 3-13.

Mofjeld, H. O. and Lavelle, J. W. 1984. Setting the length scale in a second-order closure model of the unstratified bottom boundary layer. $J$. Phys. Oceanogr. 14, 833-839.

Obukhov, A. M. 1959. Description of turbulence in terms of Langrangian variables. Adt in Geophysics 6, $113-116$

Okubo, A. 1971. Oceanic diffusion diagrams. Deep-Sea Res. 18, 789-802.

Omstedt, A. Sahlberg, J. and Svensson, U. 1983. Measured and numerically simulated autumn cooling in the Bay of Bothnia. Tellus 35A, 231-240.

Pasquill, F. and Smith, F. B. 1983. Atmospheric diffusion (3rd edition) New York: J. Wiley, $437 \mathrm{pp}$.

Pollard, R. T., Rhines, P. B. and Thompson, R. O. R. Y. 1973. The deepening of the wind-mixed layer. Geophysical Fluid Dynamics 3, 381-404.

Rahm, L. and Svensson, U. 1986. Dispersion of marked fluid elements in a turbulent Ekman layer. J. Phys. Oceanogr. 16, 2084-2096.
Richards, K. J. 1982. Modeling the benthic boundary layer. J. Geophys. Res. 12, 428-439.

Richards, K. J. 1984. The interaction between the bottom mixed layer and mesoscale motions of the ocean: A numerical study. J. Phys. Oceanogr. 14, 754-768.

Richards, K. J. 1985. The benthic boundary layer. In: I. C. R. Hunt (ed.): Turbulence and diffusion in stable entironments. The Institute of Mathematics and its Applications, Conference Series. Oxford: Clarendon Press, 237-251.

Rodi, W. 1980. Turbulence models and their applications in hydraulics. Int. Assoc. Hydrol. Res., Delft, Netherlands, 1-104.

Saffman, P. G. 1962. The effect of wind shear on horizontal spread from an instantaneous ground source. Quart. J. R. Met. Soc. 88, 382-393.

Sarmiento, J. 1978. A study of the mixing of the deep sea based on STD, radon-222 and radium-228 measurements. Ph.D. Thesis, Columbia University.

Svensson, U. 1978. A mathematical model of the seasonal thermocline. Ph.D. Thesis, Rep. 1002, Dep. Water Resour., Univ. Lund, Sweden, 187 pp.

Svensson, U. 1979. The structure of the turbulent Ekman layer. Tellus 31, 340-350.

Svensson, U. 1980. On the numerical prediction of vertical turbulent exchange in stratified flows. Proceedings, 2nd International Symposium on Stratified Flow's. The Norwegian Institute of Technology, Trondheim, Norway, 686-696.

Taylor, G. I. 1954. The dispersion of matter in turbulent flow through a pipe. Proc. Roy. Soc. A223, 446-468.

Tennekes, H. and Lumley, J. L. 1972. A first course in turbulence. Cambridge, Mass: MIT Press, $300 \mathrm{pp}$.

Thomson, D. J. 1984. Random walk modelling of diffusion in inhomogeneous turbulence. Quart. J. Roy. Met. Soc. $11 D, 1107-1120$.

Wyngaard, J. C. 1975. Modeling the planetery boundary layer -... Extension to the stable case. Boundary Layer Meteorology 9, $441-460$. 